\title{
Probe Into Abnormality of Brain Gray Matter Volumes in Schizophrenia Patients and Their Healthy Siblings
}

\author{
Chang Liu ${ }^{1^{*}}$, Zhiming $\mathrm{Xue}^{2}$, Weidan $\mathrm{Pu}^{3}$ \\ ${ }^{1}$ Brain Hospital of Hunan Province, Changsha, Hunan 410007, China; \\ ${ }^{2}$ Mental Health Institute, Second Xiangya Hospital, Central South University, \\ Changsha, Hunan 410011, China; \\ ${ }^{3}$ Medical Psychological Institute, Second Xiangya Hospital, Central South \\ University, Changsha, Hunan 410011, China.
}

*Corresponding Author: Chang Liu. Email: liuchang19861121@163. com.

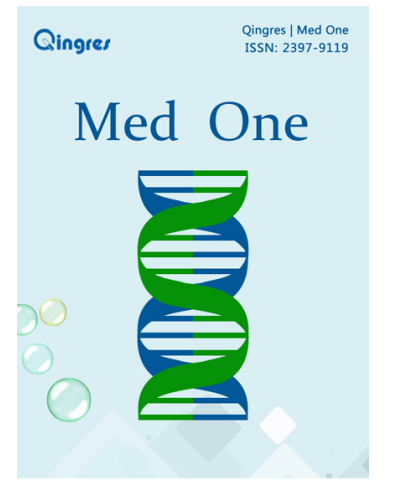

http://mo.qingres.com

\section{GOPEN ACCESS}

DOI: 10.20900/mo.201600013

Received: March 15, 2016

Accepted: May 18, 2016

Published: June 25, 2016

Copyright: $\odot 2016$ Cain et al. This is an open access article distributed under the terms of the Creative Commons Attribution License,which permits unrestricted use, distribution, and reproduction in any medium, provided the original author and source are credited.

\begin{abstract}
Objective: To investigate gray matter volume differences between schizophrenia patients, their unaffected siblings and healthy controls, and explore possible neurophysiological endophenotypes for schizophrenia.
\end{abstract}

Methods: Three-dimensional, structural magnetic resonance imaging scans of 27 schizophrenia patients, 26 unaffected siblings, and 27 healthy controls were performed. Voxel-based morphometry (VBM) was used to investigate the gray matter volume of three groups.

Results: Unaffected siblings of schizophrenics, when compared to healthy controls, showed significantly increased gray matter in the left middle occipital gyrus, precuneus, and right thalamus. Patients with schizophrenia had significantly decreased gray matter in the left middle temporal gyrus, superior frontal gyrus, and bilateral inferior temporal gyrus, and increased gray matter in the right thalamus. Schizophrenics showed gray matter decreases in the left superior frontal gyrus and the bilateral inferior temporal gyrus compared to unaffected siblings. There was a significantly negative correlation between the level of decreased gray matter in the left superior frontal gyrus, and the total PANSS score and positive syndrome score.

Conclusion: Reduced gray matter region volume observed in unaffected siblings may reflect a compensatory role and provide 
resilience to schizophrenia, while specific gray matter abnormalities of schizophrenics may be related to the illness itself.

Key Words: Schizophrenia; Sibling; Gray Matter Volume

\section{INTRODUCTION}

Schizophrenia usually occurs in young adult aged 18-25 years. It is a chronic mental illness characterized by disordered thinking, emotions and behaviors, and failure to comply with internal and external environments. It is considered a functional mental illness as there are no organic changes. Changes of the study of brain structures of living organisms allowed by the development of neuroimaging technology, allows further investigation into changes in the brain and nerve systems of schizophrenia patients. After nearly two decades of research and exploration, the results of neuroimaging studies confirm that schizophrenia is not a purely functional disease. The results of brain structure imaging studies confirms that there are differences in the morphology and brain structures of schizophrenia patients. More direct evidence, involving the abnormal brain structures of schizophrenia patients, have come from voxel-based morphology comparative studies. These studies showed reduced gray matter volumes of prefrontal lobe, superior temporal gyrus, hippocampus, and the amygdala in schizophrenia patients, as well as lateral ventricle and third ventricle enlargement ${ }^{[1,2]}$. Prior studies also confirmed that gray matter loss in the prefrontal cortex and the superior temporal gyrus cortex exists in first-episode, and chronic schizophrenics ${ }^{[3]}$. Different neuropathological changes in the brains of schizophrenia patients including cortical atrophy, enlarged ventricles, reduced amygdala and hippocampus volumes, structural disorders, hypothalamic cell loss, and volume reduction were also found in many autopsy studies.

Schizophrenia is commonly considered to be a neurodevelopmental mental illness ${ }^{[4]}$. Such abnormal neurodevelopment is thought to occur during the process of trimming and correcting synapses and regulating neurogenesis during an individual's adolescence and early adulthood. This may expose problems previously hidden or caused by genetics, environment, or interactions so as to result in schizophrenia ${ }^{[5]}$. Schizophrenia shows neurodevelopment defects, so an abnormal brain structure might exist in healthy groups who have a common genetic background and environment with schizophrenia patients. Approximately $50 \%$ of all alleles are the same between the schizophrenia patients and their unaffected siblings (brothers or sisters).

After confounding factors such as biological changes caused by antipsychotic drugs, chronic diseases, and anxiety and depression, as well as potential neurotoxic effects of mental disease are excluded, many studies of healthy siblings of schizophrenia patients showed that they suffer a loss of brain gray matter ${ }^{[6,7]}$.

These studies showed the pathological brain structure changes existed before psychiatric symptoms emeged. As of the date of this paper, no studies have examined for abnormal brain structures in unaffected siblings of schizophrenia patients. In this study, a cross-sectional comparison of early schizophrenia patients, unaffected siblings (healthy siblings with the same biological parents as the included patients) and healthy volunteers was made using a voxel-based morphology analysis to examine for gray brain matter abnormalities in unaffected siblings (relative to normal control).

It aims to discuss gray matter volume abnormalities: 1) in which brain area may relate to a genetic susceptibility to schizophrenia; 2) in which brain area may be characteristic of the disease; and, 3 ) in which brain area might be associated with a compensatory protective effect for healthy siblings of the brain area gray matter abnormality related to its genetic susceptibility.

\section{SUBJECTS AND METHODS}

\subsection{Subjects}

\subsubsection{Schizophrenic Patients (SCZ)}

The MRI data, and general clinical information, for 27 schizophrenia patients was collected for this study. All schizophrenia patients were inpatients and outpatients of the Department of Psychiatry, Second Xiangya Hospital of Central South University between September 2010 to September 2013. All schizophrenia subjects were assessed 
using the Structured Clinical Interview for DSMIV (Patient version, SCID-I/P) of the Diagnostic and Statistical Manual of Mental Disorders ${ }^{[8]}$. Inclusion criteria included i) compliance with DSMIV diagnosis criteria for schizophrenia, ii) more than one unaffected sibling for each patient who were brothers, or sisters, of the same parents, iii) 18-45 years old with a disease course of less than 5 years, iv) of Han Nationality, dextromanuality, v) no history of nerve system disease or other major physical disease, vi) no history of abuse, or reliance upon, alcohol or substances, vii) no history of electric shock treatment, viii) no use of any medication within six hours prior to imaging scanning, ix) no contraindication against MRI tests and no brain abnormalities after the MRI scanning test, and $x$ ) comprehension of the research contents, desire to participate in, and be able to complete, the entire experiment, and a signed Informed Consent.

\subsubsection{Healthy Siblings (SIB)}

Twenty-six healthy siblings were collected in this study. They were either brothers, or sisters, of the same biological parents of schizophrenics. The inclusion criteria of the healthy siblings are identical to that of the schizophrenia patients that meet the DSM-IV schizophrenia diagnosis criteria. The axis I disorders for all healthy siblings were excluded using SCID-NP.

\subsubsection{Healthy Controls ( $\mathrm{HC})$}

Twenty-seven healthy controls were collected in this study and they voluntarily enrolled through public recruitment. They were from communities in Changsha and the surrounding area. In addition to meeting inclusion criteria including items iii) $\mathrm{x}$ ), all healthy controls were to have no history of mental disease, neurological, or psychotic disorders or family disease, severe physical diseases or drug abuse. They must not have had any major psychological trauma, and their first-degree relatives were to have no history of mental disorder. The axis I disorder of all healthy controls was excluded using SCID-NP; a family history of mental diseases of firstdegree relatives was excluded using Family History Research Diagnostic Criteria (FHRDC). All subjects (Ss) were informed of the risks and benefits before the tests and executed an informed consent form. This study was approved by the Medical Research Ethics Committee of the Second Xiangya Hospital of Central South University.

\subsection{Methods}

\subsubsection{Acquisition of Structural MRI Data}

Structural MRI imaging data was acquired at the Second Xiangya Hospital of Central South University by an experienced, professional technical staff members of the Radiology Department. A Signa Twinspeed 1.5T dual-gradient magnetic resonance imaging system (General Electric, Fairfield, Connecticut, USA) was used to scan in a standardized head coil. No cognitive tasks were added during scanning. The subject lay on their back with eyes closed and was instructed to remain motionless, and try not to think about any particular person or thing. Earplugs were used against ambient noise, and a supportive foam pad was used to limit head movements. Scanning parameters included repetition time TR (12 ms), echo time TE $(4.2 \mathrm{~ms})$, flip angle (15 degrees), field of view $(24 \times 24 \mathrm{~cm})$, matrix $(512 \times 512)$, slice thickness $(1.8 \mathrm{~mm})$, gap $(0$ $\mathrm{mm})$, and 170 slices.

\subsubsection{Acquisition of Clinical Data}

An old field inventory was used to assess subject handedness. Demographic and basic clinical data for the subjects included age, gender, and educational level. The psychiatric syndrome, and severity, of the schizophrenia patients was assessed using the Positive and Negative Syndrome Scale (PANSS) ${ }^{[9]}$. PANSS was used to assess a subject's positive, negative, and general psychiatric syndromes in order to obtain a total score and factor scores, e.g. Points $\mathrm{P}, \mathrm{N}$ and $\mathrm{G}$, and record the diagnosis and disease course of the patients. Schizophrenia patient disease course averaged $18.32 \pm 15.84$ months. The disease course for 23 patients was $<2$ years, for 3 patients was $<3$ years, and for one patient was $<5$ years. All were early onset. There were 15 patients who were unmedicated, 6 who were taking risperidone (2-5 $\mathrm{mg}), 1$ was taking clozapine $(200 \mathrm{mg}), 3$ were taking quetiapine (400-600), and 2 were taking sulpiride (100-300 mg).

\subsubsection{Processing and Analysis of Structural MRI Data}

Structural MRI data were analyzed with MATLAB 7.8 \& SPM8 (http://www.fil.ion.ucl.ac.uk/spm). Structural MRI data were pre-processed with sub-toolkit VBM8 of SPM8, including standardization, partitioning, adjustment, and translation, and other steps, to obtain calibrated images of brain gray, and white, 
matter, which represents the gray matter, and white matter, volumes, respectively. The specific steps were: i) original T1 images for all subjects were subjected to Montreal Space Standardization; ii) the standardized brain structural images were partitioned into three sections, including gray matter (GM), white matter (WM), and cerebrospinal fluid (CSF); iii) the average gray matter template for all subjects was fabricated using DDARTEL; iv) partitioned brain images for all subjects were standardized, with a voxel size of $1.5 \times 1.5 \times 1.5 \mathrm{~mm} 3$; and $\mathrm{v}$ ) isotropic 8 $\mathrm{mm}$ (full-width at half-maximum, FWHM) Gaussian kerneling was used to translate the images of the three groups to obtain the high signal-noise ratio and reduce individual differences. A comparison of gray matter volumes of the translated images .

\subsubsection{Statistical Analysis}

The statistical analysis of structural MRI data was carried out using SPM8. The one-way analysis of covariance (ANCOVA) was used to compare and count significant differences in gray matter volumes among schizophrenia patients, healthy siblings, and healthy controls. The threshold value of significant difference was set as $p<0.001$. The significant result was taken from the one-way ANCOVA as a mask, and gray matter volumes for the samples of the three groups were compared with a doublesample t test model, the threshold value, of which, was set at $p<0.05$. The differences of clinical variables between two groups, or among three groups were compared with the double-sample t test model, or a one-way ANCOVA based on SPSS16.0. The correlation coefficient between gray matter volume and clinical variables was calculated using Pearson correlation analysis and a threshold value of significant difference $(p<0.05)$.

\section{RESULTS}

\subsection{Demographic Characteristics}

Demographic data and clinical characteristics for schizophrenia patients, their healthy siblings, and healthy controls is detailed in Table 1. No significant differences were found in gender, age and educational level among three groups.

Table 1. Demographic Data and Clinical Characteristics of Schizophrenia Patients, Healthy Siblings, and Healthy Controls (Mean \pm SD)

\begin{tabular}{|c|c|c|c|c|c|}
\hline \multirow{2}{*}{ Variable } & \multirow{2}{*}{$\begin{array}{l}\text { Schizophrenia } \\
\text { Patients(n=27) }\end{array}$} & \multirow{2}{*}{$\begin{array}{l}\text { Healthy } \\
\text { Siblings } \\
(n=26)\end{array}$} & \multirow{2}{*}{$\begin{array}{c}\text { Healthy } \\
\text { Controls }(n=27)\end{array}$} & \multicolumn{2}{|c|}{ Statistical Analysis } \\
\hline & & & & $F / X^{2}$ & $p$ \\
\hline Age(year) & $25.44 \pm 5.92$ & $25.56 \pm 6.44$ & $27.44 \pm 7.24$ & 0.793 & 0.696 \\
\hline Educational years(year) & $12.30 \pm 2.73$ & $12.70 \pm 2.73$ & $12.96 \pm 3.39$ & 0.365 & 0.456 \\
\hline Gender(Male/ Female) & $15 / 12$ & $16 / 11$ & $18 / 9$ & 0.713 & 0.700 \\
\hline Disease Course (month) & $18.32 \pm 15.84$ & - & - & & \\
\hline Total score (PANSS) & $85.78 \pm 12.80$ & - & - & & \\
\hline Total positive score (PANSS) & $21.56 \pm 4.92$ & - & - & & \\
\hline Total negative score (PANSS) & $23.15 \pm 5.75$ & - & - & & \\
\hline $\begin{array}{l}\text { Total score for general mental } \\
\text { disease (PANSS) }\end{array}$ & $41.30 \pm 6.38$ & - & - & & \\
\hline Disease (PANSS) & & & & & \\
\hline
\end{tabular}


Table 2 Brain Areas with Differences in Brain Gray Matter Volumes for Three Groups shown in ANCOVA

\begin{tabular}{|c|c|c|c|c|c|c|c|}
\hline \multirow{2}{*}{ Brain Area } & \multirow{2}{*}{$\begin{array}{l}\text { Voxel } \\
\text { number }\end{array}$} & \multicolumn{3}{|c|}{ MNI Coordinate } & \multicolumn{2}{|c|}{ Statistical Analysis } & \multirow{2}{*}{$\begin{array}{l}\text { Effect Size } \\
\text { Cohen's d }\end{array}$} \\
\hline & & $\mathbf{x}$ & $Y$ & $\mathbf{z}$ & $\mathbf{F}$ & $\begin{array}{l}\text { PFWE, } \\
\text { corrected }\end{array}$ & \\
\hline Middle Temporal Gyrus & 296 & -50 & -10 & -23 & 12.59 & 0.346 & 1.24 \\
\hline $\begin{array}{l}\text { Superior Temporal } \\
\text { Gyrus }\end{array}$ & 39 & -23 & -4 & 58 & 10.36 & 0.870 & 1.03 \\
\hline Inferior temporal gyrus & 39 & 50 & -46 & -23 & 9.44 & 0.958 & 1.18 \\
\hline Mid-occipital gyri & 89 & -56 & -54 & -11 & 10.36 & 0.828 & 1.45 \\
\hline Thalamus & 50 & 18 & -18 & 15 & 8.76 & 0.993 & \\
\hline
\end{tabular}

Note: MNI refers to the 3D coordinate positioning system of human brain developed by Montreal Neurological Institute (MNI). The coordinate zero is located in the center of the brain. Axis $x$ represents left/right direction and itsvalue is from a negative value to a positive value and means the direction from the leftmost to rightmost part of the human brain. Axis $y$ represents the front/rear direction and its value is from a negative value to a positive value and means the direction from the most significant end to the rearmost end of the brain. Axis z represents up/down direction. Its value is from a negative value to a positive value and means the direction from the top end to the lowest end of the brain; and the same below.

\subsection{Comparison of Whole Brain Gray Matter Volumes}

The one-way ANCOVA results showed that brain areas with differences in gray matter volumes were primarily distributed in the bilateral inferior temporal gyrus, the right thalamus, and the left superior temporal gyrus (Table 2). Post-hoc comparisons showed that the gray matter volume of the bilateral inferior temporal gyrus, the left superior temporal gyrus, and the left middle temporal gyrus of the schizophrenia patients was reduced and the gray matter volume of the right thalamus increased (Table
3, Fig. 1) compared to healthy controls. The gray matter volume of the left middle temporal gyrus, the left precuneus, and the right thalamus of healthy siblings increased (Table 4, Fig. 1) relative to the schizophrenia patients. The gray matter volume of the left superior temporal gyrus and the bilateral inferior temporal gyrus of schizophrenia patients was reduced (Table 5, Fig. 1) relative to the healthy siblings. 
Table 3. Comparison Results of Gray Matter Volumes between Schizophrenia Patients and Healthy Controls

\begin{tabular}{|c|c|c|c|c|c|c|c|}
\hline \multirow{2}{*}{ Brain Area } & \multirow{2}{*}{$\begin{array}{l}\text { Voxel } \\
\text { Number }\end{array}$} & \multicolumn{3}{|c|}{ MNI Coordinate } & \multicolumn{2}{|c|}{ Statistical Analysis } & \multirow{2}{*}{$\begin{array}{l}\text { Effect Size } \\
\text { Cohen's d }\end{array}$} \\
\hline & & $\mathbf{x}$ & $\mathbf{Y}$ & $\mathbf{Z}$ & $\mathbf{t}$ & $p$ & \\
\hline \multicolumn{8}{|c|}{ Healthy Control > Schizophrenia Patients } \\
\hline Middle Temporal Gyrus & 296 & -50 & -10 & -23 & 4.85 & 0.000384 & 1.24 \\
\hline Superior Temporal Gyrus & 11 & -21 & -4 & 58 & 3.68 & 0.013 & 1.03 \\
\hline Inferior Temporal Gyrus & 10 & 50 & -43 & -24 & 3.64 & 0.014 & 1.18 \\
\hline Inferior Temporal Gyrus & 14 & -56 & -54 & -11 & 3.58 & 0.017 & 1.45 \\
\hline \multicolumn{8}{|c|}{ Healthy Controls < Schizophrenia Patients } \\
\hline Thalamus & 50 & 20 & -21 & 16 & 3.94 & 0.06 & 2.31 \\
\hline
\end{tabular}

Table 4. Comparison Results of Gray Matter Volumes between Schizophrenia Healthy Siblings and Healthy Controls

\begin{tabular}{|c|c|c|c|c|c|c|c|}
\hline \multirow{2}{*}{ Brain Area } & \multirow{2}{*}{$\begin{array}{l}\text { Voxel } \\
\text { Number }\end{array}$} & \multicolumn{3}{|c|}{ MNI Coordinate } & \multicolumn{2}{|c|}{ Statistical Analysis } & \multirow{2}{*}{$\begin{array}{l}\text { Effect Size } \\
\text { Cohen's d }\end{array}$} \\
\hline & & $\mathbf{x}$ & $\mathbf{Y}$ & $\mathbf{Z}$ & $\mathbf{t}$ & $\begin{array}{c}\text { PFWE, } \\
\text { corrected }\end{array}$ & \\
\hline \multicolumn{8}{|c|}{ Healthy Controls < Healthy Siblings } \\
\hline Middle Temporal Gyrus & 89 & -24 & -81 & 39 & 4.31 & 0.002 & 1.24 \\
\hline Thalamus & 41 & 17 & -19 & 15 & 4.17 & 0.003 & 1.03 \\
\hline Precuneus & 22 & -17 & -63 & 39 & 4.11 & 0.004 & 1.18 \\
\hline
\end{tabular}


Table 5. Comparison Result of Gray Matter Volumes between Schizophrenia Patients and Healthy Siblings

\begin{tabular}{|c|c|c|c|c|c|c|c|}
\hline \multirow{2}{*}{ Brain Area } & \multirow{2}{*}{$\begin{array}{l}\text { Voxel } \\
\text { Number }\end{array}$} & \multicolumn{3}{|c|}{ MNI Coordinate } & \multicolumn{2}{|c|}{ Statistical Analysis } & \multirow{2}{*}{$\begin{array}{l}\text { Effect Size } \\
\text { Cohen's d }\end{array}$} \\
\hline & & $\mathbf{x}$ & $\mathbf{Y}$ & $\mathbf{Z}$ & $\mathbf{t}$ & $\begin{array}{l}\text { PFWE, } \\
\text { corrected }\end{array}$ & \\
\hline \multicolumn{8}{|c|}{ Schizophrenia Patients $<$ Healthy Siblings } \\
\hline Middle Temporal Gyrus & 39 & -24 & -6 & 60 & 4.35 & 0.002 & 1.24 \\
\hline Thalamus & 39 & 50 & -46 & -23 & 4.15 & 0.003 & 1.03 \\
\hline Precuneus & 14 & -56 & -54 & -9 & 4.15 & 0.003 & 1.18 \\
\hline
\end{tabular}

\section{SIB}
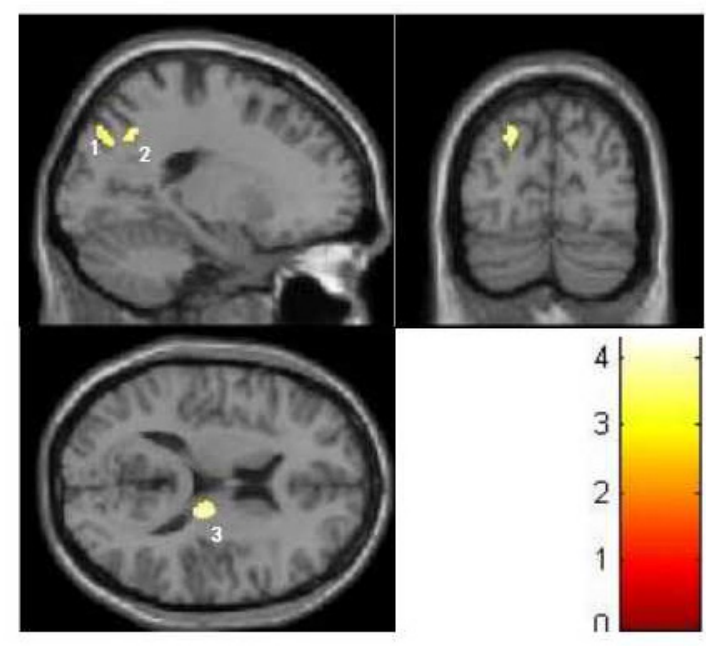

SCZ

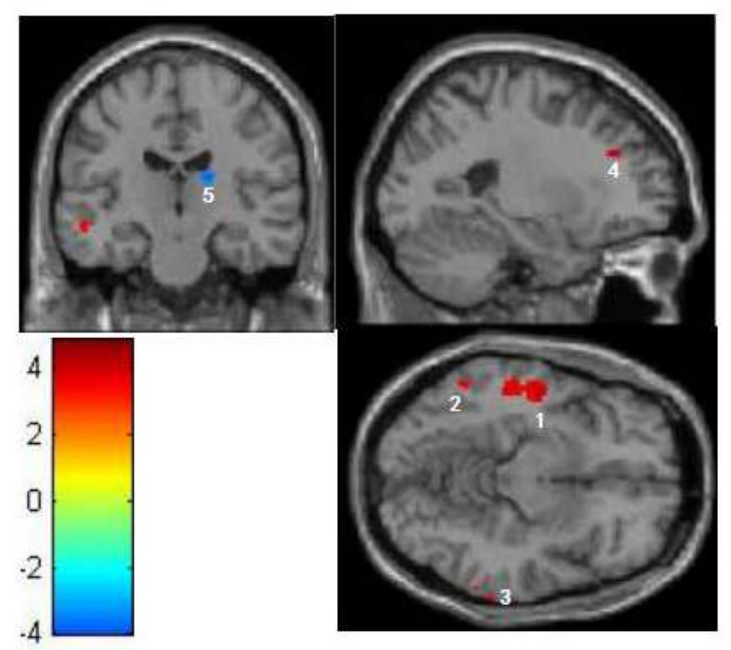

Fig. 1 Difference of gray matter volumes between healthy siblings and healthy controls \& between schizophrenia patients and healthy controls.

Notes: The left half portion in the Fig. 1 represents three brain areas with the difference of gray matter volumes between healthy siblings and healthy controls: 1 represents the left middle temporal gyrus, 2 represents the left precuneus, and 3 represents the right thalamus. The right half portion in the figure represents 5 brain areas with the difference of gray matter volumes between schizophrenia patients and healthy controls:
1 represents the left middle temporal gyrus, 2 represents the left inferior temporal gyrus, 3 represents the right inferior temporal gyrus, 4 represents the left superior temporal gyrus and 5 represents the right thalamus. Attached Figure Brain Area with Difference of Gray Matter Volumes among Schizophrenia Patients and Healthy Siblings and Healthy Controls. 


\subsection{Correlation Analysis}

The correlation analyses of schizophrenia group showed that the gray matter volume of the left superior temporal gyrus significantly negatively correlated with PANSS total score $(r=-8.99, p<0.05)$ and $p$ total score $(r=-3.54, p<0.05)$.

\section{DISCUSSION}

\subsection{Abnormal Brain Gray Matter Volume of Schizophrenia Patients}

The results show that the gray matter volume of the left superior temporal gyrus, the bilateral inferior temporal gyrus, and the left middle temporal gyrus of schizophrenia patients was reduced relative to that of the healthy controls. Gray matter volumes losses in these areas indicate that the gray matter volume reduction in schizophrenia patients mainly occurs in the front temporal lobe. This is consistent with the results of prior studies. Gray matter volumes losses exist in both acute, and chronic, schizophrenia patients and it presents mainly in the temporal, and frontal, lobes ${ }^{[7.10 .11]}$. The meta-analysis results of a structural MRI study showed that gray matter volume reductions exist in many areas of schizophrenia patients, including the superior temporal gyrus, the inferior temporal gyrus, the middle temporal gyrus, the anterior cingulated, the medial temporal lobe, the insular cortex, the hippocampus, and the parahippocampal gyrus ${ }^{[10]}$. Studies of brain structural MRIs of first-attack schizophrenia patients confirm that left middle temporal gyrus might be an early biological marker for schizophrenia ${ }^{[12]}$, and relatively consistent brain imaging evidence has proven that structural abnormalities ${ }^{[6]}$ and function ${ }^{[13,14]}$ of the middle temporal gyrus exist in schizophrenia patients. The left middle temporal gyrus is mainly responsible for processing language ${ }^{[15]}$ and concepts and the thought formation ${ }^{[16]}$. Some studies have confirmed that the inferior temporal gyrus plays an important role in schizophrenia pathogenesis. Related analyses also show that gray matter volume reduction in the inferior temporal gyrus is significantly related to the psychotic syndrome severity of schizophrenia, e.g. the severity of positive syndrome. The most common schizophrenia syndromes include auditory hallucinations, delusions, and thought disorders. Comparative structural MRI studies show that a reduction of gray matter volume of the middle temporal gyrus of schizophrenia patients. A recent morphological study has confirmed that gray matter volume reduction in the middle temporal gyrus of schizophrenia patients is significantly related to the severity of thought disorders. In the functional MRI tests of schizophrenia patients, excessive metabolism occurred in the left temporal lobe of schizophrenics. It was associated with the severity of reality distortion symptoms (hallucinations and delusions) ${ }^{[17]}$. Task-related fMRI studies showed that the reduction of functional connection of temporal lobe and frontal cortex of schizophrenia patients is associated with auditory hallucinations ${ }^{[18]}$. A recent fMRI study of language fluency tasks showed that an abnormal activation is present in the middle temporal gyrus of schizophrenics and is significantly related to the severity of thought disorders ${ }^{[13]}$. The positive syndrome of schizophrenia patients is associated with the morphological and functional abnormalities of the temporal lobes and frontal lobes. Brain structure, and brain function, studes have suggested that the middle temporal gyrus may be involved in the neuropathological changes of schizophrenia. The reduction of gray matter volume of the middle temporal gyrus may an important pathologic basis for understanding the positive syndrome of schizophrenia patients.

This study shows an enlargement of gray matter volume of the right thalamus apart from the reduction of gray matter volume of the left superior temporal gyrus, the bilateral inferior temporal gyrus, and the left middle temporal gyrus in schizophrenia patients. This study result is inconsistent with the results of prior studies. After summarizing the brain structure MRI study results of schizophrenia patients using VBM approach, it was found that past schizophreniarelated structural MRI studies, for the most part, reported that schizophrenia patient thalamus gray matter volume was significantly lower than healthy controls $^{[19,20]}$. There are some studies that report the enlargement of thalamus gray matter volume ${ }^{[21]}$. Such results may be associated with the heterogeneity of patient samples of this study, one example would be the use of antipsychotics by subjects in this study. Other points warrant further explaination.

Some studies comparing gray matter volumes of administered schizophrenia patients and nonadministered patients ${ }^{[21]}$ report thalamus volume decreases in first-attack schizophrenia patients and but increases in administered schizophrenia patients.

In this study, two thirds of $2 / 3$ the patients were taking antipsychotic medications. These antipsychotic medications may lead brain enlargement. 


\subsection{Abnormality of Brain Gray Matter Volume of Healthy Siblings}

Prior studies of unaffected siblings have reported that the gray matter volume of schizophrenia patients was significantly lower than that of the healthy controls, however, there are other studies showing the enlargement of gray matter volume in schizophrenia patients.

A possible reason for these apparently conflicting results may be that many of the healthy siblings included in those studies were in an age cohort with a high incidence of schizophrenia onset (18-25 years old), and may have had a higher genetic predisposition.

The damage to their brain gray matter was basically the same as that of schizophrenics and is reflected in gray matter volume decrease of important brain areas, including frontal lobe, temporal lobe, anterior cingulate, insular cortex, and hippocampus. The ages of healthy siblings in this study vary more around the period of schizophrenia onset (average age: $25.56 \pm 6.44$ years old). Compared to the normal controls, the morphologic abnormalities reflected of the siblings are gray matter volume enlargement, particularly in the left middle temporal gyrus, the left precuneus, and the right thalamus.

Functional MRI studies of healthy siblings who were beyond the prime onset-age for schizophrenia suggests ${ }^{[23]}$ an enhancement functional connection with brain areas related schizophrenia onset. The prior study reports that schizophrenia patients, and their healthy siblings who are at the prime-onset age for schizophrenia showed a reduction in functional connections of these brain areas.

The results of a follow-up study suggest ${ }^{[24]}$ that an identical reduction of functional connections to the areas of the brain identical to the gray matter loss of schizophrenia. This suggests a corresponding dynamic change process in the unaffected siblings. This mainly reflects in the gradual normalization of gray matter volumes of cerebral cortex areas in the unaffected siblings in their adulthood. In the highincidence period, these areas however represent the loss of brain gray matter volumes similar to the schizophrenia. These areas of the brain are mostly on the left side and relatively consistent with the "lateralization" abnormality of schizophrenia.

Researchers speculate ${ }^{[25]}$ that the dynamic process of "changing abnormality of gray matter volume into normality" can be viewed as a compensation-related change. The gray matter volume abnormalities emerge in the high-onset period, and may simply be a state-dependent change. Presently, a growing number of researchers believe that schizophrenia is a neurodevelopmental disorder. The main reasons for gray matter volume decreases in schizophrenic patients includes: excessive trimming of asymmetric synapses; decreased inhibitory pathway precision; and, nervebranch trimming. One result can be prefrontal cortex excitatory-inhibitory balance disorder out of which psychiatric symptoms emerge. The high- risk of onset period for schizophrenia is consistent with the development period of important senior cognitive brain areas as well as brain areas related to cortical connection. For schizophrenia patients this period may result in onset of psychiatric symptoms due to the neurodevelopmental disorders. Their healthy siblings, at this high-risk of onset age for schizophrenia, are subject to brain structure change similar to that of their schizophrenic siblings.

After this high-risk age bracket, gray matter volume enlargement in healthy siblings may play an important compensatory protection role and spare them from the disease.

\section{FUNDING}

National Natural Science Fund of China (program numbers: 81401125, 81171287)

\section{REFERENCES}

1. Glahn DC, Laird AR, Ellison-Wright I, Thelen SM, Robinson JL, Lancaster JL, Bullmore E, Fox PT. Meta-analysis of gray matter anomalies in schizophrenia: application of anatomic likelihood estimation and network analysis. Biol Psychiatry. 2008; 64(9): 774-781.

2. Fornito A, Yücel M, Patti J, Wood SJ, Pantelis C. Mapping grey matter reductions in schizophrenia: an anatomical likelihood estimation analysis of voxel-based morphometry studies. Schizophr Res. 2009; 108(1): 104-113. 
3. Glahn DC, Laird AR, Thelen SM, Bullmore $\mathrm{E}$. The anatomy of first-episode and chronic schizophrenia: an anatomical likelihood estimation meta-analysis. Am J Psychiatry. 2008; 165(8): 1015-1023.

4. Weinberger DR. Implications of normal brain development for the pathogenesis of schizophrenia. Arch Gen Psychiatry. 1987; 44(7): 660-669.

5. Fatemi SH, Folsom TD. The neurodevelopmental hypothesis of schizophrenia, revisited. Schizophr Bull. 2009. sbn187.

6. Hu M, Li J, Eyler L, Guo X, Wei Q, Tang J, Liu F, He Z, Li L, Jin H, Liu Z, Wang J, Liu F, Chen $\mathrm{H}$, Zhao J. Decreased left middle temporal gyrus volume in antipsychotic drug-naive, firstepisode schizophrenia patients and their healthy unaffected siblings. Schizophr Res. 2013; 144(1): 37-42.

7. Honea RA, Meyer-Lindenberg A, Hobbs KB, Pezawas L, Mattay VS, Egan MF, Verchinski B, Passingham RE, Weinberger DR, Callicott JH. Is gray matter volume an intermediate phenotype for schizophrenia? A voxel-based morphometry study of patients with schizophrenia and their healthy siblings. Biol Psychiatry. 2008; 63(5): 465-474.

8. First M, Gibbon M, Spitzer RL, Williams J. User's guide for the structured clinical interview for DSM-IV axis I Disorders (Research version). New York: Biometrics Research Department, New York State Psychiatric Institute. 1990.

9. Kay SR, Flszbein A, Opfer LA. The positive and negative syndrome scale (PANSS) for schizophrenia. Schizophr Bull. 1987; 13(2): 261.

10. Honea R, Crow TJ, Passingham D, Mackay CE. Regional deficits in brain volume in schizophrenia: a meta-analysis of voxel-based morphometry studies. Am J Psych. 2005; 162(12): 2233-2245.

11. Onitsuka T, Shenton ME, Salisbury DF, Dickey CC, Kasai K, Toner SK, Frumin M, Kikinis R, Jolesz FA, McCarley RW. Middle and inferior temporal gyrus gray matter volume abnormalities in chronic schizophrenia: an MRI study. Am J Psychiatry. 2004; 161(9): 1603.

12. Kuroki N, Shenton ME, Salisbury DF, Hirayasu $Y$, Onitsuka T, Ersner-Hershfield $\mathrm{H}$, Yurgelun-Todd D, Kikinis R, Jolesz FA, McCarley RW . Middle and inferior temporal gyrus gray matter volume abnormalities in first episode schizophrenia: an MRI study. Am J Psychiatry. 2006; 163(12):

\section{3-2110.}

13. Li X, Branch CA, Nierenberg J, DeLisi LE. Disturbed functional connectivity of cortical activation during semantic discrimination in patients with schizophrenia and subjects at genetic high-risk. Brain Imag Behav. 2010; 4(1): 109-120.

14. Yu R, Chien YL, Wang HLS, Liu CM, Liu CC, Hwang TJ, Hsieh MH, Hwu HG, Tseng W. Frequency-specific alternations in the amplitude of low-frequency fluctuations in schizophrenia. Human Brain Map. 2014; 35(2): 627-637.

15. Kiehl KA, Smith AM, Mendrek A, Forster BB, Hare RD, Liddle PF. Temporal lobe abnormalities in semantic processing by criminal psychopaths as revealed by functional magnetic resonance imaging. Psychiatry Res. 2004; 130(1): 27-42.

16. Wei T, Liang X, He Y, Zang Y, Han Z, Caramazza $\mathrm{A}, \mathrm{Bi}$ Y. Predicting conceptual processing capacity from spontaneous neuronal activity of the left middle temporal gyrus. J Neurosci. 2012; 32(2): 481-489.

17. Kaplan RD, Szechtman H, Franco S, Cleghorn JM. Three clinical syndromes of schizophrenia in untreated subjects: relation to brain glucose activity measured by position emission tomography (PET). Schizophrenia Res. 1993; 11(1): 47-54.

18. Lawrie SM, Buechel C, Whalley HC. Frith CD, Friston KJ, Johnstone EC. Reduced frontotemporal functional connectivity in schizophrenia associated with auditory hallucinations. Biol Psychiatry. 2002; 51(12): 1008-1011.

19. Portas CM, Goldstein JM, Shenton ME, Hokama $\mathrm{HH}$, Wible CG, Fischer I, Kikinis R, Donnino R, Jolesz FA, McCarley RW. Volumetric evaluation of the thalamus in schizophrenic male patients using magnetic resonance imaging. Biol Psychiatry. 1998; 43(9): 649-659.

20. Csernansky JG, Schindler MK, Splinter NR, Wang L, Gado M, Selemon L, Rastogi-Cruz D, Posener JA, Thompson PA, Miller M. Abnormalities of thalamic volume and shape in schizophrenia. Am J Psychiatry. 2004; 161(5): 896-902.

21. Gur RE, Maany V, Mozley PD, Swanson C, Bilker W, Gur RC. Subcortical MRI volumes in neuroleptic-naive and treated patients with schizophrenia. Am J Psychiatry. 1998; 155(12): 1711-1717. 
22. Borgwardt SJ, Riecher-Rössler A, Dazzan P, Chitnis X, Aston J, Drewe M, Gschwandtner U, Haller S, Pfluger M, Rechsteiner E, D'Souza M, Stieglitz RD, Radu EW, McGuire PK. Regional gray matter volume abnormalities in the at risk mental state. Biol Psychiatry. 2007; 61(10): 1148-1156.

23. Wang J, Cao H, Liao Y, Liu W, Tan L, Tang Y, Chen J, Xu X, Li H, Luo C, Liu C, Merikangas KR, Calhoun V, Tang J, Shugart Y, Chen X. Three dysconnectivity patterns in treatmentresistant schizophrenia patients and their unaffected siblings. Neurolmage Clin. 2015; 8:
95-103.

24. Gogtay N, Greenstein D, Lenane M, Clasen L, Sharp W, Gochman P, Butler P, Evans A, Rapoport J. Cortical brain development in nonpsychotic siblings of patients with childhoodonset schizophrenia. Arch Gen Psychiatry. 2007; 64(7): 772-780.

25. Gogtay N, Vyas NS, Testa R, Wood SJ, Pantelis C. Age of onset of schizophrenia: perspectives from structural neuroimaging studies. Schizophr Bull. 2011; 37(3): 504-513. 